\title{
Measurement of Credit Risk of Small and Medium-sized S\&T Enterprises in China
}

\author{
Jia-wen Zhang ${ }^{1}$, Long-hui Chen ${ }^{1}$, Xiang-yun Liu ${ }^{1} \&$ Fen Ding ${ }^{1}$ \\ ${ }^{1}$ Finance Department, Guangdong University of Finance and Economics, Guangzhou, China \\ Correspondence: Fen Ding, Finance Department, Guangdong University of Finance and Economics, 21 Chisha Road, \\ Guangzhou 510320, Guangdong, China. E-mail: finody168@163.com
}

Received: April 20, 2014

Accepted: May 16, 2014

Online Published: June 23, 2014

doi:10.5430/ijba.v5n4p21

URL: http://dx.doi.org/10.5430/ijba.v5n4p21

\begin{abstract}
This paper mainly studies the measurement of credit risk of Chinese small and medium-sized enterprises in Science and Technology (SMEs in S\&T). Starting from the characteristics of the development of S\&T enterprises, this paper selects the chinext 12 Chinese small enterprises annual data as sample, and builds a first-passage-time jump-diffusion structural model to measure small and mid-sized enterprise credit risk on the basis of the traditional KMV model.At last,it concludes that the first-passage-time jump-diffusion structural model has higher accuracy on the measurement of the credit risk for it obeys to the high risk and high volatility of the small and medium-sized S\&T enterprises. At the same time, it puts forward the policy proposal on the development of the small and medium-sized S\&T enterprises in China.
\end{abstract}

Keywords: small and medium-sized S\&T enterprise, credit risk, jump-diffusion process

\section{Introduction}

\subsection{Research Background}

SMEs in S\&T have spring up and develop fast in China, which become the most dynamic innovators in the technical progress. Until the end of 2011, the number is more than 70000, which accounts for $18.22 \%$ of the total number of the SMEs. The gross annual income coming from technology and trading is more than 800 billion RMB, accounting for $21.57 \%$ of sales revenue with the same caliber. Compared the year of 2012 with 1992, the technology gross income, gross profit, taxes, export of SMEs in S\&T have respectively experienced an increase of 50 times, 30 times, 46 times and 70 times, respectively.

Their rapid development boosts up the demand for loans. However, compared with the large firms, SMEs have more barriers to financing. Financial institutions have low willingness to extent credit because of high risks and costs of lending to SMEs in S\&T. So difficulties in financing becomes the primary factor that restricts the development of SMEs in S\&T, for banks and other financial institutions can not evaluate the loan's credit risks about these companies accurately. Finally, this paper will represent a new model to measure the credit risk of the SMEs.

\subsection{Research Significance}

Many researches have focused on practices of MNCs and neglected the operate way of SMEs. The finance of SMEs in S\&T has the following characteristics:

- In the early period, SMEs in S\&T need large amount of funds, which mainly rely on external financing.

- The main external funds' channel is bank loans, but banks just approve liquid funds and pledged loans in renewal of fixed assets, rather than long-term loans, which could hinder the rapid development of SMEs in S\&T.

- The primary factor is the scale of assets that decides whether the company can get loans or not. But SMEs in S\&T usually don't have enough loan collaterals, and they mainly own intangible properties that are difficult to evaluate.

Due to these characteristics, the successful rate of applying loans in SMEs of S\&T is very low. At the same time, traditional evaluation model can barely reflect the real value of SMEs in S\&T. Therefore, on the basis of real options model, this paper builds the first-passage-time jump-diffusion structural model which is fit for credit risk assessment 
of loans for the SMEs in S\&T, and tries to properly describe the enterprises' loans and R\&D projects' cash flow, in order to get a better way to evaluate their credit risks.

\subsection{Literature Review}

Approving loans to SME of S\&T, banks face two major risks: the credit risks from SME of S\&T and the market risks of loan programs. In this study, we only discuss the credit risks. Foreign scholars begun to study credit risks assessment earlier, and the techniques about it can be broadly classified into two categories:

1) The traditional credit risks assessment methods

The traditional credit risks assessment methods which contain expert systems, rating systems and credit scoring models: Expert systems are all qualitative approaches, which take the ability, reputation and prospects for the development of the whole industry of the loan's owner and administer into consideration; Rating systems are very professional assessing methods, which are done by professional credit rating companies; Credit scoring models are most widely used in these traditional methods, Beaver(1966) considered single factor to measure enterprises' credit risks. Edward Altman (1968) developed a multiple regression method and constructed Z-score model, which was very popular in commercial banks in United States in the last century and obtained higher successful rate in assessing credit risks of the customers of enterprise class. Ohlson (1980) tried to use more generous assumption to build logistic model that many factors selected as input variables, such as corporate profitability, management capacity, etc. The development of computer technology and mathematical statistics tools let more and more artificial intelligence rating models emerge, such as decision trees, neural networks, etc. What's more, some researches like Prater, Edmund,Ghosh, Soumen(2005) described the distinct differences between the small and large firms operating in Europe and found that there more barriers for SMEs to develop or globalize.

On the other hand, the domestic scholars have done researches about loans pricing model which can assess credit risks indirectly through pricing loans. There have already been three main categories: the summarizing cost models, the benchmark rate plus point pricing models, the customer profitability analysis models. Currently, these three loan pricing models have been widely applied in state-owned commercial bank.

2) The modern credit risks assessment models

In this kind of models, the basic one is VAR model based on the value distribution, of which the typical one is JP Morgan's Credit Metrics model (1997), and the core of this model is the transition probability matrix of credit risk rating. The KMV company built a KMV model (Crosbie, 1997) on the basis of Merton model, which exploited time series of the company's stock price to deduce the distribution of credit risks. The research institutions, Petr GAPKO and Martin SMID doubted the KMV model's three assumptions (factor normalized distribution, the fixed values of lost given default (LGD) and static model) and adopted some fat tails factor to build a dynamic stochastic models of stochastic LGD in multiple times, the empirical results showed that this model can prevent banks from the huge unexpected credit losses. DonL. Mcleish and Adam Metzler (2011) introduced the first-passage-time model into Brownian motion to predict credit risks, and took the prediction of common systematic risks into the model. Donatien Hainaut \& Olivier Le Courtois (2013) develop a switching regime version of the intensity model for credit risk pricing which is well suited to model changes of volatility trends in credit spreads, related to modifications of unobservable economic factors.

Although domestic researches about credit risks model started late, in recent years many articles and empirical researches about credit risk measurement have been springing up. Many domestic scholars did numerous researches about modern models from the outside academic realm, especially the KMV model. Han Liyan and Zheng Chengli (2005) pointed out input data and forecast results's vague expressions of the KMV model getting closer to facts, which were more complying with the request of decisions. This KMV default predicted model also has some defects like fixed default point, which could be better to use fuzzy stochastic methods to explore the company's default prediction on the basis of KMV model. Zhang Ling and Yang Zhenshi (2004) calculated sample companies'deflaut distance on three deflaut point value, they did t-test and Wilcoxon rank sum test with the default distance of matching sample. They considered that when the model's default point value was equal to current liabilities, the model has the strongest resolution for listed companies. Hong Kong scholars Cho-Hoi Hui, et al (2012) also amended one assumption of KMV model--a static capital structure, and proposed a time-varying dynamic capital structure. Empirical studies showed that in credit risks prediction, the dynamic capital structure was a very critical factor.

In brief, currently the number of researches about the credit risks measurement in SME of S\&T is very limited. As we all know, enormous funds demand is the biggest financing characteristics of SME of S\&T, but this kind of loans 
contain huge risks and high uncertainty which are likely to lead to an underestimation of their credit risks.

Therefore, in practice, we should promote the construction of technology finance index and index system about loan projects for SME of S\&T, providing a reliable basis for making lending decisions. This paper recommends the first-passage-time jump-diffusion structural model which has a higher accuracy on the measurement of the credit risk and gives some suggestions about the development of the SMEs in China.

\section{Model Construction}

\subsection{Data and Sample}

Due to the short term from the startup of GEM(less than four years), the listed time of many SME of S\&T is less than one year in China. To ensure complete data we need, this study selects ones with more than two years listed time as our sample. Owing to different funds demand and development situation of different industries, this paper takes full consideration about each industries' characteristics on sample selection, in order to compare credit risks of SME of $\mathrm{S} \& \mathrm{~T}$ in different industries. The selected sample is as follows:

Table 1. The sample of SMEs in S\&T from GEM

\begin{tabular}{llll}
\hline Industy name & SMEs in S\&T 1 & SMEs in S\&T 2 & SMEs in S\&T 3 \\
\hline BioI & AKSW (300009) & BLYY (300016) & HRYY (300026) \\
CEII & CTRJ (300036) & HLCT (300045) & TRS (300229) \\
PEMI & LPYL (300003) & YPYL (300030) & LBYQ (300206) \\
EEMI & OBT (300053) & DFRS (300118) & ZHCC (300224)
\end{tabular}

Notes: The sample period: 1/1/2012 to 31/12/2012; Data Source: wind

In the Table 1, we separately choose three typical SMEs of S\&T from the biopharmaceutical industry (BioI), the computer electronic information industry (CEII), the professional equipment manufacturing industry(PEMI) and the electronic equipment manufacturing industry(EEMI)

\subsection{The Model Basic Hypothesis}

The basic hypothesis in the first-passage-time jump-diffusion structural model are as follows:

- The assets of SMEs in S\&T consist of owners' equity and liabilities, and the liabilities can be divided into current liabilities and long-term liabilities.

- The stocks' value in GEM of SMEs in S\&T represents the equity value of this company

- The assets of SMEs in S\&T are highly uncertain. As time goes by, their value keep fluctuating. When the assets' value is more than liabilities, the company will pay back the debts. Otherwise, the company will choose default

- The volatilities of stocks on GEM can represent the volatilities of equities in SMEs of S\&T .Because SMEs of S\&T are accompanied by swift growth as well as high risks, their shares' price usually fluctuates dramatically, which could influence their assets' value. In this study, we construct the model based on their high volatility

- The company can default when its assets' value below a certain value in credit period, rather than in the end of credit period.

\subsection{Determination of Parameters}

- Research time in this model $(T)$. Set $T$ be one year, which only considers credit risks of SMEs in S\&T in one year.

- Risk-free rate in period $T(\mathrm{r})$. Set $r$ be one-year deposit interest rate of 3\% from the People's Bank of China.

- The strike price $(X) . X$ equals to current liabilities $(C L)$ plus long- term liabilities $(L L)$ from SMEs of S\&T:

$$
X=C L+L L
$$

- Default point $(D P) . D P$ is set to take the general form in the KMV model: 


$$
D P=C L+0.5 \times L L
$$

- Lowest limit in the first-time-passage model $(D)$. In theory, $D$ 's value is much lower than $D P$ 's, Set $D$ denote as current liabilities:

$$
D=C L
$$

- Return of security $i$ in period T( $\left.\varphi_{i}\right) . \varphi_{i}$ equals to the sum of daily yield in period $T$.

$$
\begin{gathered}
\varphi_{i}=\sum\left(\begin{array}{ll}
\ln & p_{t} / p_{t-1}
\end{array}\right) \\
p_{t=\text { Closing price of security } i \text { on day } \mathrm{t}}
\end{gathered}
$$

The yearly price volatility of stocks $\left(\delta_{E}\right)$. We can build GARCH model to get predicted volatility of the second day after the observation period, then turn this volatility into volatility per year:

$$
\delta_{E}=\delta_{d a y} \times \sqrt{252}
$$

- The market value of equity $(E i)$. There exists tradable shares and non-tradable shares in China' stock market. But in this study, the price of non-tradable shares is equal to the price of tradable ones:

$$
E=e \times p_{t}
$$

$\mathrm{e}=$ the total number of equities

- The intensity of Poisson Jump ( ${ }^{\lambda}$ ). There are two reasons that can bring saltatory risks to the companies: Technical progress, renewal of facility from the companies themselves, and technical innovation from

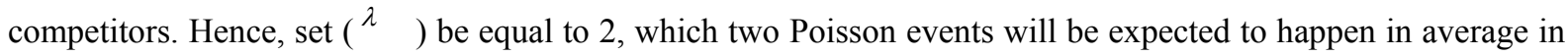
a unit time.

- The amplitude of Poisson Jump $\left(\xi_{k}\right)$. Let $\ln \left(\xi_{k}+1\right)$ be subject to the independent gaussian distribution.

2.4 The Construction of Jump-Diffusion Stochastic Process

Owing to the characteristic of SMEs of S\&T in GEM, we construct this stochastic process with multriple poisson jump-diffusion, so that

$$
\begin{aligned}
d S_{t} & =S_{t-}\left[(\mu-\lambda v) d t+\sigma d W_{t}+d Y_{t}\right] \\
& =S_{t-}\left[(\mu-\lambda v) d t+\sigma d W_{t}+\xi_{k} d N_{t}\right]
\end{aligned}
$$

$S_{t}=$ asset prices, $W_{t}$ is denoted as wiener process under the possibility of $\mathrm{P}, \mu=$ the drift rate of asset prices, $Y_{t}$ represents multriple poisson process, $N_{t}$ is subject to poisson process whose parameter is $\lambda$.

We assume that $S, \mathrm{~N}$ and $\mathrm{Y}$ are right continuous. Let $t_{k}$ be the time when the jump k happen in poisson process, so at the moment of $t_{k}$. 


$$
\begin{gathered}
\Delta Y_{t_{k}}=Y_{t_{k}}-Y_{t_{k^{-}}}=\xi_{k} \\
\Delta S_{t_{k}}=S_{t_{k}}-S_{t_{k^{-}}}=S_{t_{k^{-}}} \Delta Y_{t_{k}}=S_{t_{k^{-}}} \xi_{k} \Rightarrow S_{t_{k}}=S_{t_{k^{-}}}\left(\xi_{k}+1\right)
\end{gathered}
$$

In equation (9), $\xi_{k} \geq 1$ and $\ln \left(\xi_{k}+1\right) \sim N\left(\theta, \sigma^{2}\right)$

Given presented parameters, according to Ito's lemma, we can get the explicit solution

$$
S_{t}=S_{0} \exp \left\{\left(\mu-\frac{1}{2} \sigma^{2}-\lambda v\right) t+\sigma W_{t}\right\} \prod_{k=1}^{N}\left(\xi_{k}+1\right) t \in[0, \infty)
$$

\subsection{The Calculation of Assets' Value and Volatility}

According to real option theory, we can get

$$
\begin{aligned}
E\left(0, S_{t}\right) & =E^{Q}\left[e^{-r T}\left(S_{T}-X\right)^{+}\right] \\
& =E^{Q}\left[e^{-r T} S_{T} I_{\left\{S_{T} \geq X\right\}}\right]-X E^{Q}\left[e^{-r T} I_{\left\{S_{T} \geq X\right\}}\right]=G-Z
\end{aligned}
$$

In equation (11), $\mathrm{X}=$ strike price, $\mathrm{T}=$ =maturity date, $\left.{ }^{I}{ }^{*}\right)$ is a discriminant function, which means that when $S_{T} \geq X$, the value of $I\left\{s_{T} \geq X\right\}$ is one, otherwise, zero.

According to non-arbitrage pricing theory under the equivalent martingale measure, we can gain the $\mathrm{G}$ in equation (11), combined with equation (10).

$$
G=E^{Q}\left[e^{-r T} S_{T} I_{\left(S_{T} \geq X\right)}\right] S_{T}=S_{0} \exp \left(\left(\mathrm{r}-\frac{1}{2} \sigma^{2}-\lambda v\right) T+\sigma W_{T}\right) \prod_{k=1}^{N_{T}}\left(\xi_{k}+1\right) I_{\left\{S_{T \geq} X\right.}
$$

In equation (12), $\mathrm{L}=\delta W_{T}, \mathrm{M}=\sum_{k}^{N}{ }^{T}{ }_{1} \quad \mathrm{n} \quad\left(\xi_{k}+1\right)$

When $N_{T}=n,(L+M) \sim N\left(n \theta, \delta^{2} T+n \delta^{2}\right)$.

Due to relevant theory (Note 1), we can get the final form of $G$

$$
G=E^{Q}\left[e^{-r T} S_{T} I_{\left\{S_{T} \geq X\right\}}\right]=S_{0} e^{-\left(\frac{1}{2} \sigma^{2}+\lambda v\right) T} \sum_{n=0}^{\infty} \frac{e^{-\lambda T}(\lambda T)^{n}}{n !} e^{n \theta+\frac{\sigma^{2} T+n \delta^{2}}{2}} N\left(d_{1}\right)
$$

In equation (13), $d_{1}=\frac{\ln \frac{S_{0}(1+v)^{n}}{X}+(r-\lambda v) T+\frac{1}{2}\left(\sigma^{2} T+n \delta{ }^{2}\right)}{\sqrt{\sigma^{2} T+n \delta^{2}}}$

$\mathrm{N}(*)$ is a standard normal cumulative probability distribution.

Also, we can get the $Z$ in equation (11)

$$
Z=X e^{-r t} \sum_{n=0}^{\infty} \frac{e^{-\lambda T}(\lambda T)^{n}}{n !} N\left(d_{2}\right)
$$

In equation (14), $d_{2}=d_{1}-\sqrt{\delta^{2} T+n \delta^{2}}$

In summary, we can get the real option's pricing formula which is subject to Jump-diffusion stochastic process. 


$$
E=S_{0} e^{-\left(\frac{1}{2} \sigma^{2}+\lambda v\right) T} \sum_{n=0}^{\infty} \frac{e^{-\lambda T}(\lambda T)^{n}}{n !} e^{n \theta+\frac{\sigma^{2} T+n \delta^{2}}{2}} N\left(d_{1}\right)-X e^{-r t} \sum_{n=0}^{\infty} \frac{e^{-\lambda T}(\lambda T)^{n}}{n !} N\left(d_{2}\right)
$$

Then according to Ito's lemma, we can know the relation between the volatility of asset price and the volatility of stock price:

$$
\begin{aligned}
& \sigma_{E} E=S_{0} \sigma_{s} \frac{\partial E}{\partial S}=S_{0} \sigma_{s} e^{-\left(\frac{1}{2} \sigma^{2}+\lambda v\right) T} \sum_{n=0}^{\infty} \frac{e^{-\lambda T}(\lambda T)^{n}}{n !} e^{n \theta+\frac{\sigma^{2} T+n \delta^{2}}{2}} N\left(d_{1}\right) \\
& \left.\sigma_{E} E=S_{0} \sigma_{s} \frac{\partial E}{\partial S}=S_{0} \sigma_{s}\left(\sum_{n=0}^{\infty} \frac{[\lambda(1+v) T]^{n} e^{-\lambda(1+v) T}}{n !}\right) N\left(d_{1}\right)\right) \\
& E\left(\xi_{k}\right)
\end{aligned}
$$

$$
\text { In equation (17), } d_{1}=\frac{\ln \frac{S_{0}(1+v)^{n}}{X}+(r-\lambda v) T+\frac{1}{2}\left(\sigma^{2} T+n \delta^{2}\right)}{\sqrt{\sigma^{2} T+n \delta^{2}}}, \mathrm{~N}(*) \text { is a standard normal }
$$

cumulative probability distribution.

We can get the the market value of enterprises'assets and the volatility of assets through solving equations (17) and (15).

\subsection{Default Risks Assessment}

Now, we know the distance of default about SMEs in GEM, which is

$$
D D=\frac{E\left(S_{T}\right)-D P}{E\left(S_{T}\right) \sigma_{S}}
$$

In equation (18), $E\left(S_{T}\right)$ is the expected value of enterprises' assets. In this study, we imitate many paths of changes in asset price due to Momte Carlo stochastic method to get related assets' terminal values. Based on these figures, we can gain $E\left(S_{T}\right)$ in approximation which is equal to their average values.

Given the normal distribution of asset price in the end of term, we can gain the default probability(PD)

$$
P D=N(-D D)
$$

In equation(19), $\mathrm{N}(*)$ is a standard normal cumulative probability distribution.

\subsection{The First Passage Structural Model}

In the first passage structural model, as long as enterprise' assets are less than the lowest limit(D) at any moment before debt maturity, the company will choose defaulting.

$$
t_{D}=\inf \left\{u>t, S_{u}=D\right\}
$$

In equation (20), $t_{D}$ delegate the first time when the asset price is equal to $D$. Inf is an information set.

Actually, the essence of the first passage structural model is one kind of down-and-out European call barrier options. When the price of subject matter is lower than the appointed price, the barrier option is cancelled automatically, and becomes valueless. However, if the price always surpasses the appointed price, the option's value will not be zero.

\section{Results and Discussion}

Based on the first-passage-time jump-diffusion structural model we can get the Table 2. 
Table 2. Essential parameters about the sample data of SMEs in S\&T from GEM

\begin{tabular}{|c|c|c|c|c|c|c|c|}
\hline name & stock code & $\mathrm{E}$ & $\mathrm{X}$ & DP & $\mathrm{D}$ & $\sigma_{E}$ & $\mu_{E}$ \\
\hline AKSW & 300009 & 17.048 & 0.729 & 0.722 & 0.715 & 0.443 & 0.0190 \\
\hline BLYY & 300016 & 11.227 & 0.596 & 0.429 & 0.264 & 0.592 & 0.5392 \\
\hline HRYY & 300026 & 56.112 & 4.152 & 3.766 & 3.381 & 0.582 & 1.2241 \\
\hline CTRJ & 300036 & 13.044 & 1.700 & 1.619 & 1.538 & 0.215 & -0.5982 \\
\hline HLCT & 300045 & 34.103 & 0.685 & 0.549 & 0.412 & 0.221 & 0.1780 \\
\hline TES & 300229 & 27.968 & 0.784 & 0.766 & 0.749 & 0.864 & -0.1687 \\
\hline LPYL & 300003 & 72.836 & 1.318 & 1.260 & 1.202 & 0.409 & -0.3946 \\
\hline YPYL & 300030 & 13.409 & 0.786 & 0.725 & 0.663 & 0.153 & -0.1288 \\
\hline LBYL & 300206 & 14.577 & 0.896 & 0.677 & 0.458 & 0.200 & -0.0372 \\
\hline OBT & 300053 & 11.930 & 0.153 & 0.135 & 0.118 & 0.173 & -0.3018 \\
\hline DFRS & 300118 & 24.248 & 20.582 & 19.301 & 18.021 & 0.164 & -0.6361 \\
\hline ZHCC & 300224 & 39.096 & 4.734 & 4.691 & 4.649 & 0.535 & -0.1778 \\
\hline
\end{tabular}

Note: $u_{E}=$ the drift rate of equities

We can know from the Table 2 that enterprises in the electronic equipment manufacturing industry have more debts than the ones in other industries, especially DFRS(300118), whose total liabilities in the selected sample is the highest, equal to its own equities in the stock market. Except that, the volatility of the biopharmaceutical enterprises is higher than others, which is caused by this industry's own characteristics, so the industry risk is usually higher. In other industries, there is always a company, whose equity volatility is higher than the two other samples' in the same industry. For example, TRS (300229) in the computer electronic information industry, whose equity volatility reached a high point--0.864, indicates that among industries, the impact of these industry risk factors on enterprises' risk is not prominent.

According to the data in Table 2, combined with equations (15) and (17), we can get the assets' market value $(S)$, the volatility of assets ( $\delta_{S}$ ), and the yearly drift rate of assets $\left({ }^{u_{S}}\right)$, just as follows:

Table 3. Relevant parameters about assets of SMEs in S\&T from GEM (Note 3)

\begin{tabular}{lllll}
\hline name & stock code & $S$ & $\sigma_{S}$ & $\mu_{S}$ \\
\hline ALSW & 300009 & 17.722 & 0.427 & 0.0183 \\
BLYY & 300016 & 11.766 & 0.567 & 0.5145 \\
HRYY & 300026 & 59.774 & 0.550 & 1.1491 \\
CTRJ & 300036 & 14.495 & 0.196 & -0.5383 \\
HLCT & 300045 & 34.757 & 0.217 & 0.1747 \\
TES & 300229 & 28.689 & 0.844 & -0.1645 \\
LPYL & 300003 & 74.095 & 0.402 & -0.3879 \\
YPYL & 300030 & 14.130 & 0.146 & -0.1222 \\
LBYQ & 300206 & 15.395 & 0.190 & -0.0352 \\
OBT & 300053 & 12.078 & 0.171 & -0.2981 \\
DFRS & 300118 & 34.822 & 0.136 & -0.4429 \\
ZHCC & 300224 & 43.074 & 0.492 & -0.1612 \\
\hline
\end{tabular}

Note: $\delta_{S}=$ the volatility of assets, $u_{S}=$ the drift rate of equities. 
In Table 3, we can gain that the assets' market value $(S)$ of one company is less than the summary of the equities and liabilities, which is complied with general theories. When its liabilities account for large parts of its equities, its

$\delta_{s}$ is very different from $\delta_{E}$. When the company's liabilities is much less than its equities, its $\delta_{S}$ is close to $\delta_{E}$, which we can simplify the calculation.

According to the parameters from Table 1 and Table 2, calculating by the Momte Carlo stochastic method in statistical software R-3.0.0, we get each companies' default distance $(D D)$ in the first-passage-time jump-diffusion structural model, and compare this result with default distance from KMV model $(D D 1)$ and jump-diffusion model $(D D 2)$. We can gain another table we call table4 with default distance of SMEs in S\&T from GEM in the three models.

Table 4. Default distance of SMEs in S\&T from GEM in three models

\begin{tabular}{lllll}
\hline name & Stock code & DD & DD1 & DD2 \\
\hline AKSW & 300009 & 2.254 & 7.234 & 2.256 \\
BLYY & 300016 & 1.723 & 6.382 & 1.724 \\
HRYY & 300026 & 1.787 & 6.725 & 1.788 \\
CTRJ & 300036 & 4.16 & 7.8 & 4.161 \\
HLCT & 300045 & 4.598 & 19.724 & 4.6 \\
TES & 300229 & 1.159 & 3.645 & 1.16 \\
LPYY & 300003 & 2.431 & 8.969 & 2.432 \\
YPYY & 300030 & 6.464 & 19.432 & 6.465 \\
LBYQ & 300206 & 5.211 & 15.994 & 5.213 \\
OBT & 300053 & 5.762 & 24.251 & 5.763 \\
DFRS & 300118 & 0.395 & 1.104 & 0.954 \\
ZHCC & 300224 & 1.803 & 3.933 & 1.806 \\
\hline As & & Tabe & &
\end{tabular}

As we can see from the Table 4, the DD1 is obviously less than DD2, which means that after adding the jumping items, the jump-diffusion model becomes more accurate and more efficient in measuring listing risks in GEM. There exists a narrow gap between DD1 and DD2, and the main reason is that the liabilities of most sample enterprises are much less than their equities, and the lowest limit is so low that leads to the narrow gap. But if the total amount of enterprise's liabilities is close to its equities', the first-passage-time jump-diffusion structural model is more sensitive than the jump-diffusion model about credit risks' assessment.

Furthermore, we can gain their default probabilities(PD) in line with these default distance figures I Table 4 as in Table 5.

Table 5. Default probabilities of SMEs in S\&T from GEM in three models (Note 4)

\begin{tabular}{lllll}
\hline name & Stock code & PD & PD1 & PD2 \\
\hline ALSW & 300009 & $1.21 \%$ & 0 & $1.20 \%$ \\
BLYY & 300016 & $4.24 \%$ & 0 & $4.24 \%$ \\
HRYY & 300026 & $3.70 \%$ & 0 & $3.69 \%$ \\
CTRJ & 300036 & 0 & 0 & 0 \\
\hline
\end{tabular}




\begin{tabular}{lllll}
\hline HLCT & 300045 & 0 & 0 & 0 \\
TES & 300229 & $12.32 \%$ & $0.01 \%$ & $12.30 \%$ \\
LPYL & 300003 & $0.75 \%$ & 0 & $0.75 \%$ \\
YPYL & 300030 & 0 & 0 & 0 \\
LBYQ & 300206 & 0 & 0 & 0 \\
OBT & 300053 & 0 & 0 & 0 \\
DFRS & 300118 & $34.64 \%$ & $13.48 \%$ & $17.00 \%$ \\
ZHCC & 300224 & $3.57 \%$ & $0.00 \%$ & $3.55 \%$
\end{tabular}

When one company's liabilities' value is much less than its equities' value, and the asset volatility is high, PD2 is obviously higher than PD1. If the asset volatility is below a certain value, the default probability from the first-passage-time jump-diffusion structural model and pure jump-diffusion model is almost similar. However when there is no difference between one company's liabilities and its equities, even though the asset volatility is very low, PD2 is higher than PD1, and PD is much higher than PD2.

\section{Conclusions}

This paper starts from the own characteristics of SMEs in S\&T, and constructs the first-passage-time jump-diffusion structural model to measure enterprises' credit risks. This model have two advantages: Firstly, it overcomes one major flaw of the traditional KMV model, which allows enterprises to default in the credit period, not just the end of credit period; Secondly the increasing jumping item is added into the traditional KMV model which is in accordance with high risk, high volatility about SMEs in S\&T, and have more accuracy on measuring credit risks.

We can conclude from the empirical researches as follows:

- The equities' volatility of SMEs of S\&T in biopharmaceutial industry is universally higher than enterprises' in other industries, which is caused by this industry's own characteristics.

- When one company's liabilities' value is much less than its equities' value, one company's asset volatility is very close to its equity volatility. At the same time, if its asset volatility is high, the jump-diffusion model is more capacity of credit risk assessment than KMV model. This two models is almost similar when the asset volatility below a certain value. Also, the first-passage-time jump-diffusion structural model has few difference in jump-diffusion model.

- When there is no difference between one company's liabilities and its equities, one company's asset volatility is quite different with its equity volatility. Even though the asset volatility is very low, the first-passage-time jump-diffusion structural model is more conservative and more accurate than the other two models.

\section{Recommendations}

- Broaden direct financing channels for SMEs in S\&T. The direct financing of enterprises in developed countries accounts for $70 \%$ in total amount of financing, however, the direct financing of SMEs in S\&T only accounts for $5 \%$. As a result, the administrators should properly ease listing conditions of GEM for SMEs in S\&T, open refinancing in GEM, propel SMEs' share transfer system in the nationwide, and improve equity investment exiting mechanism.

- Innovate and enrich financial products which is fit for SMEs in S\&T. Banks and other financial institutions should change their ideas about SMEs in S\&T, firmly establish a customer-centric business philosophy, keep developing characteristic products based on SMEs of S\&T in different types and periods, and provide customized financial products and services for them.

- Consolidate information services for SMEs in S\&T. The financing difficulty in these enterprises can be solved by getting more information and improve the level of credit through the third party.

- Guide the development of the SMEs and increase political supports about financial services for SMEs in S\&T. These companies are short of capacity against risks, which increases the difficulty to get financing supports. So it is necessary to create necessary financing conditions for them to get assets through the government's caring policy. 


\section{Acknowledgements}

The authors would like to thank the anonymous reviewers and editors for their helpful comments in preparing this paper. Liu also wants to thank National Natural Science Fund program (71273067) and Chinese Education Ministry under Humanities and Social Science Planning Fund program (11YJA790089)

\section{References}

Ahmad, Kamilah. (2014). The Adoption of Management Accounting Practices in Malaysian Small and Medium-Sized Enterprises. Asian Social Science, 236-249.

Altman. (1968). Financial ratios discriminate analysis and the prediction of corporate bankruptcy. Journal of Finance, 23, 189-209.

Beaver W H. (1966). Financial Ratios as Predictors of Failure. Journal of Accounting Research, 71-111.

Bridge, J., \& Peel, M. J. (1999). Research Note: A Study of Computer Usage and Strategic Planning in the SME Sector. International Small Business Journal, 17(4), 82-87.

Caroline Hillairet, \& Ying Jiao. (2012, April). Credit risk with asymmetric information on the default threshold. Stochastics An International Journal of Probability and Stochastic Processes, 84(2-3), 183-198.

Cho-Hoi Hui, \& Tek-Chuen Wong. (2012). Does using time-varying target leverage ratios in structural credit risk models improve their accuracy?. Journal of Risk Model Validation, 27-49.

Claudio Albanese, \& Oliver X. Chen. (2005, June). Discrete credit barrier models. Quantitative Finance, 5(3), 247-256

Donald, R. Hodgman. (1960). Credit Risk and Credit Rationing. The Quarterly Journal of Economic, 74(2), 258-278.

Donatien Hainaut, \& Olivier Le Courtois. (2013). An intensity model for credit risk with switching Lévy processes. Quantitative Finance, 1-13.

DonL. Mcleish, \& Adam Metzler. (2011). A multiname first-passage model for credit risk. The Journal of Credit Risk, 35-64.

Fabio Wendling Muniz de Andrade, \& Abraham Laredo Sicsú. (2008, May). A Credit Risk Model for Consumer Loan Portfolios. Latin American Business Review, 8(3), 75-91.

Gupta, Jairaj, Gregoriou, Andros, \& Healy, Jerome. (2013, Jan). The Effect of Internationalization on Modeling Credit Risk for SMEs: Evidence from UK Market. SSRN Working Paper Series.

Han, liyan, \& Zheng, chengli. (2005). The adjusted assessment and application of financial risks' probabilities. The Journal of Shanxi university of Finance, (4), 42-49.

J. P. L. Hatchetta, \& R. Kühnb. (2009). Credit contagion and credit risk. Quantitative Finance, 9(4), 373-382.

Kundid, Ana, \& Ercegovac, Roberto. (2011). Credit rationing in financial distress: Croatia SMEs' finance approach. International Journal of Law and Management, 62-84.

Lugovskaya, Lyudmila. (2010, March). Predicting default of Russian SMEs on the basis of financial and non-financial variables. Journal of Financial Services Marketing, Special Issue: In the Grip of the Credit Crunch: 14.4, 301-313.

Nicholas Wilson, Barbara Summers, \& Robert Hope. (2000, November). Using Payment Behaviour Data for Credit Risk Modeling. International Journal of the Economics of Business, 7(3), 333-346.

Ohlson, James A. (1980). Financial Ratios and the Probabilistic Prediction of Bankruptcy. Journal of Accounting Research, (Spring), 109-131.

Özgür Arslan, \& Mehmet Baha Karan. (2009, January). Credit risks and internationalization of SMEs. Journal of

Business Economics and Management, 10(4), 361-368.

Prater, Edmund, \& Ghosh, Soumen. (2005, April). Current Operational Practices of U.S. Small and Medium-Sized Enterprises in Europe. Journal of Small Business Management, 43(2), 155-169.

Su-Lien Lu. (2013, May). Measuring credit risk by using a parameterized model under risk-neutral measure. Applied Economics Letters, 20(8), 719-723.

Thomas Henschel. (2007). Risk Management Practices in the Main Industries of German Small to Medium-Sized Enterprises: An Empirical Investigation. School of Accounting. Retrieved from 
http://researchrepository.napier.ac.uk

Tomasz R. Bielecki, Stéphane Crépey, \& Monique Jeanblanc. (2010, December). Up and down credit risk. Quantitative Finance, (10), 1137-1151.

Umberto Cherubini, \& Giovanni Della Lunga. (2001, May). Liquidity and credit risk. Applied Mathematical Finance, $8(2), 79-95$.

Viviani, Diego, Giorgino, Marco Minola, Tommaso, \& Dellarossa, Mattia. (2008). Capital structure and innovation of SME's in European countries. ICSB World Conference Proceedings, 1-49. Washington: International Council for Small business (ICSB).

Wilson, T. (1997a). Portfolio Credit Risk I. Risk, 10(a).

Wilson, T. (1997b). Portfolio Credit Risk II. Risk, 10(b).

Yong Kim. (2013, December). Modeling of commercial real estate credit risks. Quantitative Finance, 13(12), 1977-1989.

Zhang, Ling, Yang, Zhenshi, \& Chen, Shou. (2004). The application reaserch of KMV model about credit risks of listing companies. Systems Engineering, (11), 33-38.

\section{Notes}

Note 1. If $X \sim N\left(u_{X}, \delta_{X}^{2}\right), Y \sim N\left(u_{Y}, \delta_{X}^{2}\right)$, and there exists covariation between $\mathrm{X}$ and $\mathrm{Y}$ defined as $\operatorname{cov}(X, Y)$, we get $E\left(e^{X} I_{\{Y \geq b\}}\right)=e^{\mu_{X}+\frac{\sigma_{X}{ }^{2}}{2}} N\left(\frac{-b+\mu_{Y}+\operatorname{cov}(X, Y)}{\sigma_{Y}}\right), \mathrm{N}(*)$ in this equation is a standard normal cumulative probability distribution.

Note 2. If $X \sim N\left(u_{X}, \delta_{X}^{2}\right), Y \sim N\left(u_{Y}, \delta_{X}^{2}\right)$, and there exists covariation between $\mathrm{X}$ and $\mathrm{Y}$ defined as $\operatorname{cov}(X, Y)$, we get $E\left(e^{X} I_{\{Y \geq b\}}\right)=e^{\mu_{X}+\frac{\sigma_{X}{ }^{2}}{2}} N\left(\frac{-b+\mu_{Y}+\operatorname{cov}(X, Y)}{\sigma_{Y}}\right), \mathrm{N}(*)$ in this equation is a standard normal cumulative probability distribution.

Note 3. $u_{S}=$ the drift rate of equities, $\delta_{S}=$ the volatility of assets

Note 4. Zero in the table 5 means the results are so small that can be ignored.

Let PD, PD1 and PD2 separately be denoted as the default probabilities from the first-passage-time jump-diffusion model, KMV model and pure jump-diffusion model. 